\title{
Percutaneous Endovascular Salvage Techniques for Implanted Venous Access Device Dysfunction
}

\author{
Stéphane Breault • Frédéric Glauser • \\ Malik Babaker • Francesco Doenz • \\ Salah Dine Qanadli
}

Received: 31 January 2014/ Accepted: 22 June 2014/Published online: 6 September 2014

(C) Springer Science+Business Media New York and the Cardiovascular and Interventional Radiological Society of Europe (CIRSE) 2014

\begin{abstract}
Purpose Implanted venous access devices (IVADs) are often used in patients who require long-term intravenous drug administration. The most common causes of device dysfunction include occlusion by fibrin sheath and/or catheter adherence to the vessel wall. We present percutaneous endovascular salvage techniques to restore function in occluded catheters. The aim of this study was to evaluate the feasibility, safety, and efficacy of these techniques.

Methods and Materials Through a femoral or brachial venous access, a snare is used to remove fibrin sheath around the IVAD catheter tip. If device dysfunction is caused by catheter adherences to the vessel wall, a new "mechanical adhesiolysis" maneuver was performed. IVAD salvage procedures performed between 2005 and 2013 were analyzed. Data included clinical background,
\end{abstract}

S. Breault · M. Babaker · F. Doenz · S. D. Qanadli $(\bowtie)$ Diagnostic and Interventional Radiology Department, Lausanne University Hospital, Rue du Bugnon 46, 1011 Lausanne, Switzerland

e-mail: salah.qanadli@chuv.ch

S. Breault

e-mail: stephane.breault@chuv.ch

M. Babaker

e-mail: malik.babaker@chuv.ch

F. Doenz

e-mail: francesco.doenz@chuv.ch

F. Glauser

Angiology and Diagnostic and Interventional Radiology

Departments, Lausanne University Hospital, Rue du Bugnon 46,

1011 Lausanne, Switzerland

e-mail: frederic.glauser@chuv.ch catheter tip position, success rate, recurrence, and rate of complication.

Results Eighty-eight salvage procedures were performed in 80 patients, mostly women $(52.5 \%)$, with a mean age of 54 years. Only a minority $(17.5 \%)$ of evaluated catheters were located at an optimal position (i.e., cavoatrial junction $\pm 1 \mathrm{~cm})$. Mechanical adhesiolysis or other additional maneuvers were used in 21 cases ( $24 \%$ ). Overall technical success rate was $93.2 \%$. Malposition and/or vessel wall adherences were the main cause of technical failure. No complications were noted.

Conclusion These IVAD salvage techniques are safe and efficient. When a catheter is adherent to the vessel wall, mechanical adhesiolysis maneuvers allow catheter mobilization and a greater success rate with no additional risk. In patients who still require long-term use of their IVAD, these procedures can be performed safely to avoid catheter replacement.

Keywords Central venous access - Venous intervention - Endovascular treatment . Vena cava . Obstruction $\cdot$ Cancer

\section{Introduction}

Implanted venous access devices (IVADs) are often used in patients who require long-term intravenous drug administration, such as chemotherapy, parenteral nutrition, or antibiotics and can also be used for blood punctures $[1,2]$. Central catheter dysfunction is a frequent complication with a reported occlusion rate varying from 3.2 to $33 \%$ [3-6]. The most common causes of catheter occlusion include adherence to vessel wall and fibrin sheath formation around catheter tip. Patients usually present with 
Table 1 Patient characteristics

\begin{tabular}{lll}
\hline Sex $(\%)$ & $M(\%)$ & $38(47.5)$ \\
& $F(\%)$ & $42(52.5)$ \\
Age (years) & Mean (range) & $54(17-84)$ \\
Indication for IVAD (\%) & Chemotherapy & $73(91)$ \\
& $(\%)$ & \\
& Antibiotics $(\%)$ & $7(9)$ \\
Catheter indwelling time (days) & Mean (range) & $366(2-3,304)$ \\
\hline
\end{tabular}

increased resistance when injecting in the port and/or the absence of blood reflux on aspiration. First-line therapy for catheter dysfunction consists of the use of thrombolytic agents, which according to some investigators can reestablish catheter patency in a majority of cases [4, 7-9]. If thrombolytic therapy fails, catheter replacement has long been the only available solution. Percutaneous endovascular catheter salvage techniques were developed in our institution as a less invasive and more comfortable alternative to catheter replacement, thereby avoiding the risks and costs associated with surgery.

The main objective of this study was to describe and evaluate IVAD salvage procedures, including a newly described "mechanical adhesiolysis" maneuver, used to restore catheter function. We evaluate the feasibility, safety, and efficacy of these techniques.

\section{Materials and Methods}

Consecutive IVAD salvage procedures performed between October 2005 and November 2013 were analyzed. Approval from our institution's Ethical Committee was obtained, and patients gave informed consent for the procedure. Eighty patients who underwent a salvage procedure during the study period were included. Forty-two patients were women $(52.5 \%)$, and 38 were men $(47.5 \%)$; mean age was 54 years (range 17-84). All patients were referred for IVAD dysfunction, which was defined as absence of blood reflux on aspiration or high resistance at injection. Main reasons for IVAD implantation were chemotherapy administration $(n=73 ; 91 \%)$ and intravenous antibiotics $(n=7 ; 9 \%)$. Time from catheter insertion to its dysfunction was registered when available (Table 1).

\section{Catheter Evaluation and Salvage Procedures}

The procedures were performed in a day-hospital setting or during patient hospitalization. Patients were brought to the angiography suite for device evaluation where we first tested for blood aspiration. Patients were placed in supine position and images were first acquired without contrast to confirm adequate needle placement in port chamber and to make sure that the catheter itself was intact and correctly attached to the port. Using iodinated contrast media, digitally subtracted images were then acquired to look for signs of catheter/venous obstruction.

At evaluation, the presence of a fibrin sheath is characterized by a so-called pseudoenlargement of catheter on contrast injection (Fig. 1). Linear reflux of contrast can be seen around catheter along with, most of the time, a jet of contrast exiting sideways along the catheter. A fibrin sheath can also form a pouch distal to catheter tip, which can fill with contrast. Catheter adherence to the vessel wall is suspected when its tip does not move with heart motion and patient movement or if it is in close relation to the vessel wall. These elements were noted in the procedure report if observed.

Catheter tip position was evaluated in full inspiration. It was marked as optimal if located $\pm 1 \mathrm{~cm}$ from the cavoatrial junction. Malposition was divided as "primary" if the catheter was not correctly placed during implantation (e.g., catheter was too short) or as "secondary" if the catheter moved after being correctly placed at surgery. After confirmation of fibrin sheath formation and/or vessel wall adherences as the cause for catheter dysfunction, all of our patients underwent a salvage procedure either immediately at the time of evaluation or at a later time.

Under sterile conditions and after local anesthesia (Rapidocain $1 \%$; Sintetica, Mendrisio, Switzerland), femoral vein puncture was performed with or without ultrasound guidance. A $6 \mathrm{~F}, 45-60 \mathrm{~cm}$-long introducer sheath was inserted up to the right atrium and put under continuous saline perfusion to prevent thrombus formation within the sheath. Cavogram was first performed to exclude thrombus in the superior vena cava (SVC) and/or surrounding the catheter. A snare catheter, single loop (Amplatz Goose Neck; ev3 Inc., Plymouth, MN) or clover-shaped (Atrieve; Medical Device Technologies Inc., Gainesville, FL), was then used to encircle the IVAD catheter. It was pushed as high as possible around the IVAD catheter, closed, and pulled back while maintaining moderate tension (Fig. 2). This "strips" the fibrin sheath from the catheter. It can be repeated as many times as needed until catheter function is re-established.

If the catheter could not be grabbed with the snare because of malposition or vessel wall adherences, additional maneuvers similar to those previously described by Qanadli et al. [10] were used to mobilize its tip. In these situations, we used a $4 \mathrm{~F}$ high-torque pigtail catheter that we positioned up and over the IVAD catheter and pulled back along the IVAD catheter to break vessel wall adherences (Fig. 3). We named this maneuver "mechanical adhesiolysis." In harder cases, a twisting motion can be used to encircle the pigtail around the IVAD catheter before 


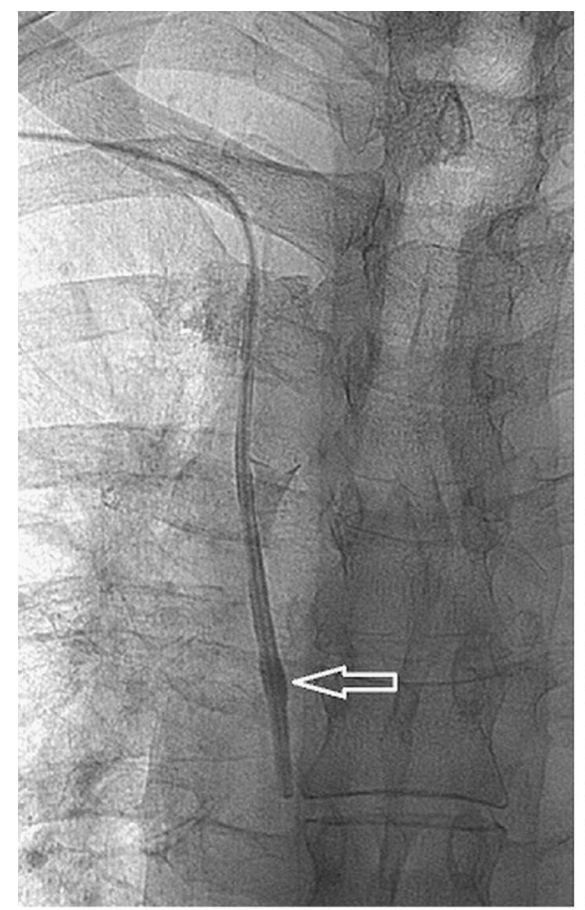

(A)

Fig. 1 Fibrin sheath around dysfunctional catheter. A This image shows the so-called pseudoenlargement sign, which is specific for fibrin sheath formation around catheter. Contrast injected in the port cannot exit at the catheter tip and refluxes around the catheter (open arrow). The catheter itself can be seen in this image as a linear hypodense structure inside the fibrin sheath. B This image also shows

pulling back (Fig. 4). Once the catheter tip was free and could be grabbed with the snare, a regular stripping technique was performed as described previously.

Puncture site was closed by manual compression and patients stayed in bed for a minimum of $3 \mathrm{~h}$ for routine observation. The length of the procedure, the material that was used, and whether the radiologist had to use mechanical adhesiolysis or other additional maneuvers was registered in the procedure report.

Technical success was defined as re-establishment of blood reflux within device port and restoration of a normal flow with a straight jet of contrast coming out at catheter tip and no resistance at injection. Complications were classified as minor or major according to the Society of Interventional Radiology standards [11].

\section{Results}

A total of 88 catheter salvage procedures were performed in 80 patients during the study period. Catheter dysfunction occurred at a mean of 366 days (range 2-3,304) after implantation. Almost 3 of 4 procedures $(n=64 ; 72.3 \%)$ were performed in a day-hospital setting in out-patients. A

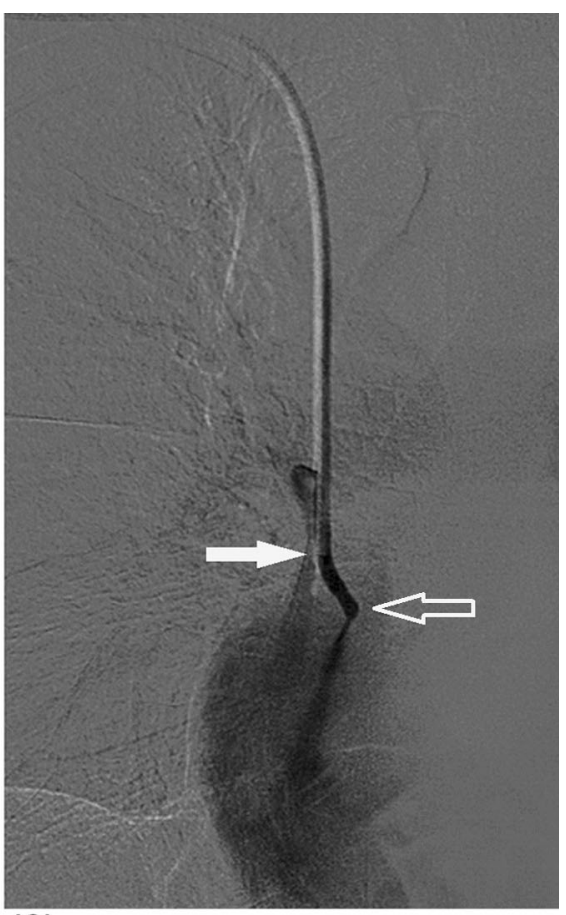

(C)

the pseudoenlargement sign (open arrow). A jet of contrast is seen exiting sideways along catheter (solid arrow). C In this third case, the fibrin sheath forms a pouch (open arrow) distal to the catheter tip (solid arrow) in the manner of a sock being pulled off a foot. In addition, note a jet of contrast exiting sideways from the pouch

little less than half of the procedures $(n=43 ; 49 \%)$ were performed immediately after device evaluation. For the remaining cases $(51 \%)$, procedure was performed after a mean of 9.5 days (range 1-77). Only $17.5 \%$ of evaluated catheters were located at optimal position, i.e., the cavoatrial junction $\pm 1 \mathrm{~cm}$ (Table 2).

Most of the procedures were performed with a snare catheter alone $(76 \%)$. Mechanical adhesiolysis or other additional maneuvers were used in 21 of 88 procedures $(24 \%)$. For those cases, the catheter tip was in a suboptimal position $>85 \%$ of times and located most commonly in the innominate vein or the upper third of SVC (Table 3). One patient presented with a secondary malposition of the catheter, which was located in the right internal jugular vein. This warranted an endovascular repositioning maneuver using a pigtail and a guidewire before stripping with a snare was possible (Fig. 5). Another patient had a very short catheter implanted with its tip positioned at the distal left brachiocephalic vein. This patient underwent two stripping attempts that were unsuccessful before having the catheter surgically replaced. Dysfunction of the newly placed catheter due to fibrin sheath warranted another stripping attempt, which was successful. In another case, the IVAD catheter was rolled up in the left subclavian vein. 
Fig. 2 Stripping maneuver using snare catheter. A A snare is used to encircle the IVAD catheter and pushed as high as possible. B It is partly closed around the catheter and pulled back while applying moderate tension to remove the fibrin sheath and restore IVAD function. Caution is advised because a snare can cut through the catheter if too much tension is applied. This procedure can be repeated as many times as necessary to re-establish catheter function
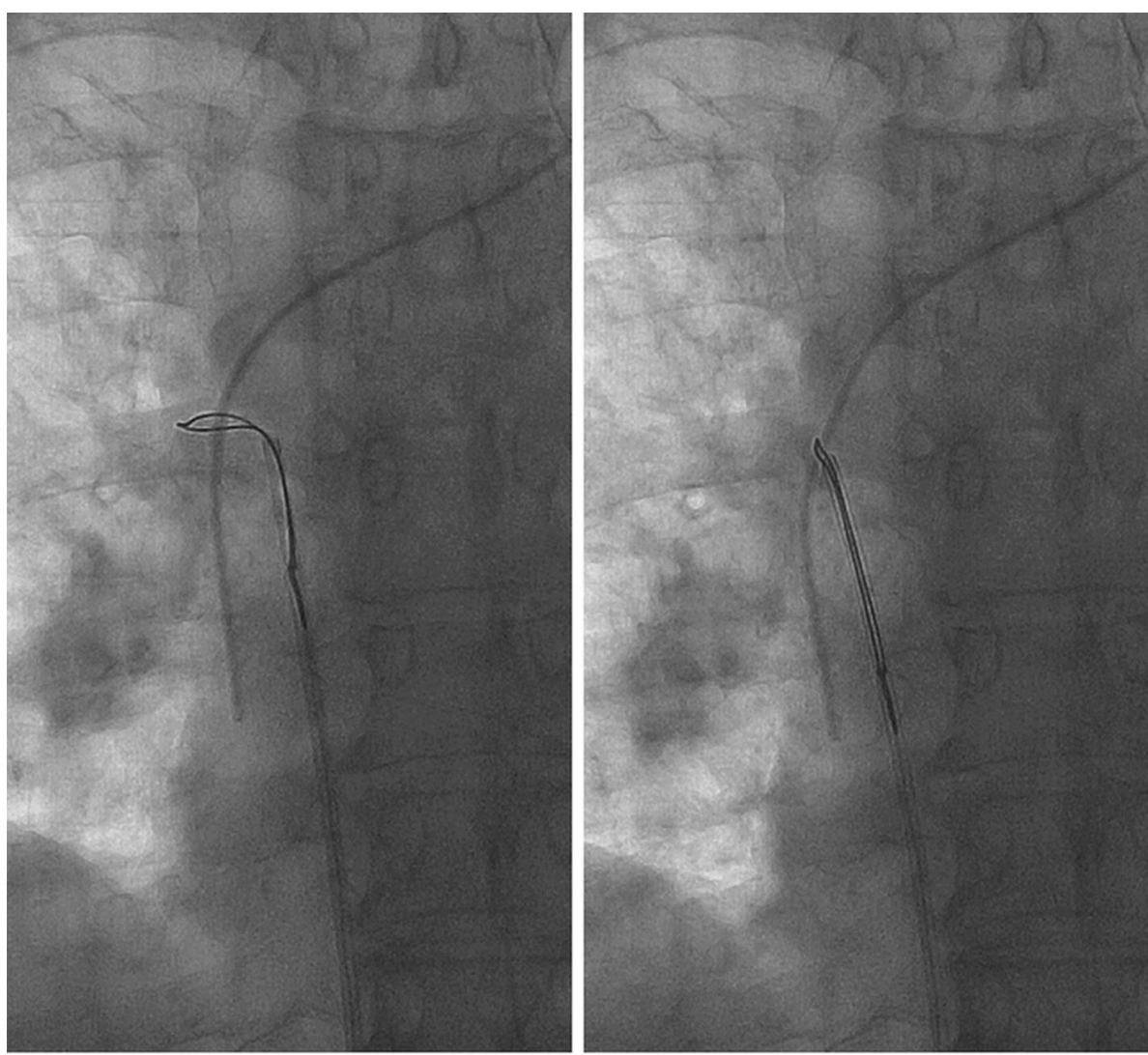

(B)
Venography showed severe stenosis of the subclavian vein proximal to the catheter, and angioplasty was performed to re-establish vein patency. Using the pigtail, we were then able to position the IVAD catheter in the SVC before performing regular stripping with a snare (Fig. 6). The majority of patients presented with nonstenosed central veins, and venoplasty or stenting was used in only 3 patients $(4 \%)$.

Global technical success rate was $93.2 \%$ for all procedures. The most commonly reported cause for technical failure was catheter tip malposition and/or adherence to the vessel wall and inability to grab catheter tip with the snare or to perform mechanical adhesiolysis.

Procedure length was available for 66 of 88 procedures. Mean time of the procedure was 48.6 min (range 16-107) if catheter salvage was performed during the same procedure as device evaluation and $27.5 \mathrm{~min}$ (range 6-102) if it was performed at a later time.

No complications directly linked to the procedure were noted.

Clinical follow-up after procedure was available for a mean time of 609 days (range 0-2,946) with patient death being the main reason for short-term follow-up. One patient presented venous thrombosis 21 days after device implantation, which was diagnosed on Doppler ultrasound.
She had her catheter surgically removed and received anticoagulation therapy. A total of eight recurrences $(9.1 \%)$ in 6 patients were noted during study period. They occurred at a mean of 272 days (range 23-498) after successful stripping. A salvage procedure was repeated in these patients, and technical success was achieved in 6 of 8 procedures. For both failed procedures, a second attempt was successful and allowed for a $100 \%$ patient-specific success rate in recurrences. No association between catheter tip position and recurrence was observed.

During the study period, 13 patients had their catheter removed due to end of treatment. This was performed after a mean of 733 days (range 21-1,363). Only two patients had their catheter surgically replaced after a failed salvage procedure $(2.5 \%)$. Three malpositioned catheters (too short) were replaced to avoid recurrence of obstruction $(3.5 \%)$.

\section{Discussion}

This study allowed for evaluation of feasibility and safety of IVAD salvage procedures, especially a newly described mechanical adhesiolysis technique using a pigtail catheter. Similar procedures have been described for treatment of 
Fig. 3 Mechanical adhesiolysis maneuver. When the IVAD catheter is adherent to the vessel wall, it must be freed before it can be grabbed with the snare. We used a $4 \mathrm{~F}$ pigtail catheter that we position up and over the IVAD catheter $(\mathbf{A})$ and pull it back along the IVAD catheter to break vessel wall adherences (B)
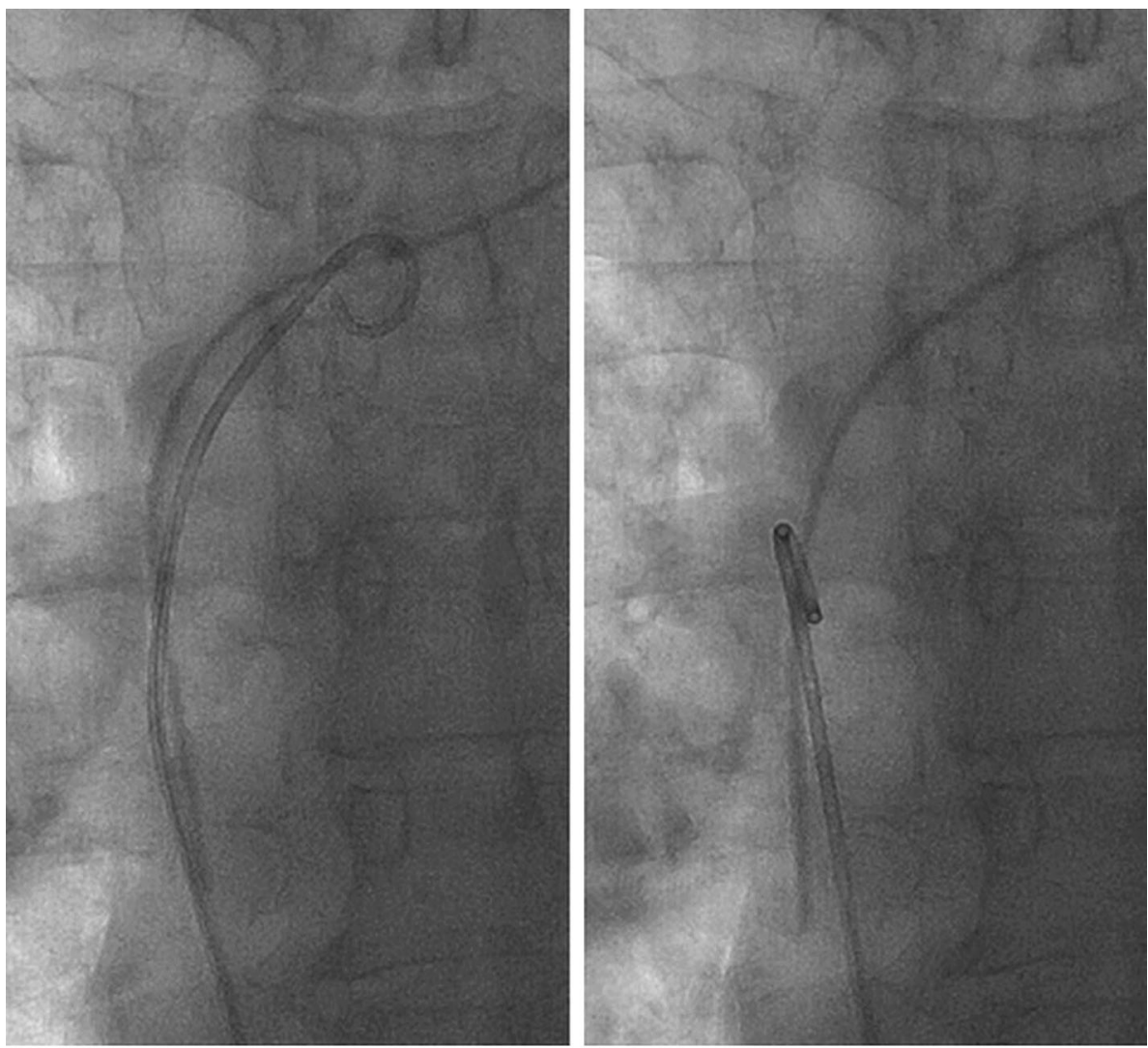

(A)

(B)

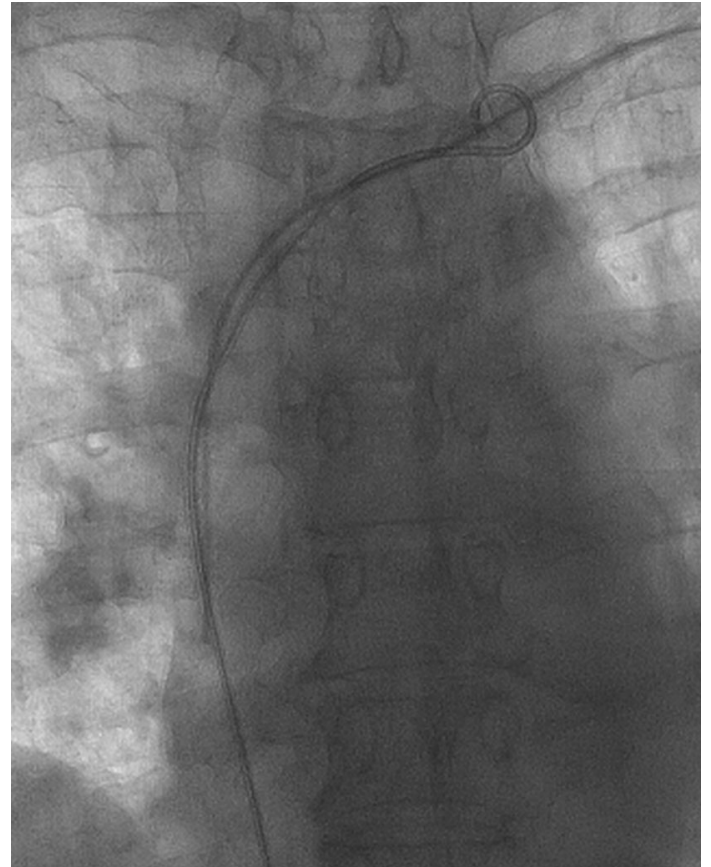

Fig. 4 Twisting motion in mechanical adhesiolysis. In difficult cases, when vessel wall adherences are hard to break, a twisting motion can be used with the pigtail catheter to encircle it around the IVAD catheter and allow for a better grasp. Different guidewires can also be placed in the pigtail to adjust stiffness as needed (not shown)
Table 2 Catheter tip position at initial evaluation

\begin{tabular}{lc}
\hline Position & $n(\%)$ \\
\hline SVC upper third & $15(18.8)$ \\
SVC mid third & $20(25.0)$ \\
SVC lower third & $17(21.3)$ \\
Cavo atrial junction $( \pm 1 \mathrm{~cm})$ & $14(17.5)$ \\
Right atrium & $8(10.0)$ \\
Innominate vein & $10(12.5)$ \\
Right internal jugular & $2(2.5)$ \\
Left subclavian & $1(1.3)$ \\
Azygos & $1(1.3)$ \\
\hline
\end{tabular}

occluded IVAD catheters as well as obstruction of dialysis catheters with high technical success and low complication rates $[8,9,11-13]$.

To our knowledge, this is the largest published series specifically in regards to IVAD catheter salvage. Only a few articles report techniques specifically dedicated to salvage IVADs. Heye et al. described 37 stripping procedures in IVADs with a success rate of $91.9 \%$ [9], which is quite similar to what we achieved. In their series, they reported the need to free the catheter from vessel wall 
Table 3 Catheter tip position when additional maneuvers such as mechanical adhesiolysis had to be used

\begin{tabular}{ll}
\hline Position & $n(\%)$ \\
\hline SVC upper third & $5(23.8)$ \\
SVC mid third & $1(4.8)$ \\
SVC lower third & $1(4.8)$ \\
Cavo atrial junction $( \pm 1 \mathrm{~cm})$ & $3(14.3)$ \\
Right atrium & $1(4.8)$ \\
Innominate vein & $6(28.6)$ \\
Right internal jugular & $2(9.5)$ \\
Left subclavian & $1(4.8)$ \\
Azygos & $1(4.8)$ \\
\hline
\end{tabular}

adherences in $44.1 \%$ of cases, which is almost twice as often as what we encountered.

In our institution, catheters are implanted by surgeons in the operating room and under fluoroscopy guidance. Catheter tip position has been reported to be a precipitating factor for catheter dysfunction [4, 14, 15]. Catheter tips that are located in the brachiocephalic vein or the upper part of SVC have been associated with a greater rate of dysfunction [14, 16, 17]. Every case in our series that required additional maneuvers, such as mechanical adhesiolysis, presented with a catheter tip located in a suboptimal position, most commonly in the innominate vein or the upper third of the SVC. This shows, as some investigators have suggested, that adequate fluoroscopy-guided implantation of IVADs by trained professionals is strongly advised to avoid catheter-related complications [2, 14, 18, 19].

Disruption of the fibrin sheath by angioplasty during an over-the-wire catheter exchange procedure is often used to restore function in a tunneled dialysis catheter [20-23]. However, this procedure cannot be used in patients with IVAD because it is impossible to place a guidewire in the IVAD catheter.

Fibrin sheath stripping using a snare has been described by other investigators and has proven to be a useful technique to restore function in occluded IVADs [9, 12, 24]. The ability to mobilize catheter tip from vessel wall is an important factor for the technical success of salvage procedures. When this cannot be achieved with the snare catheter alone, other techniques can be used. Investigators

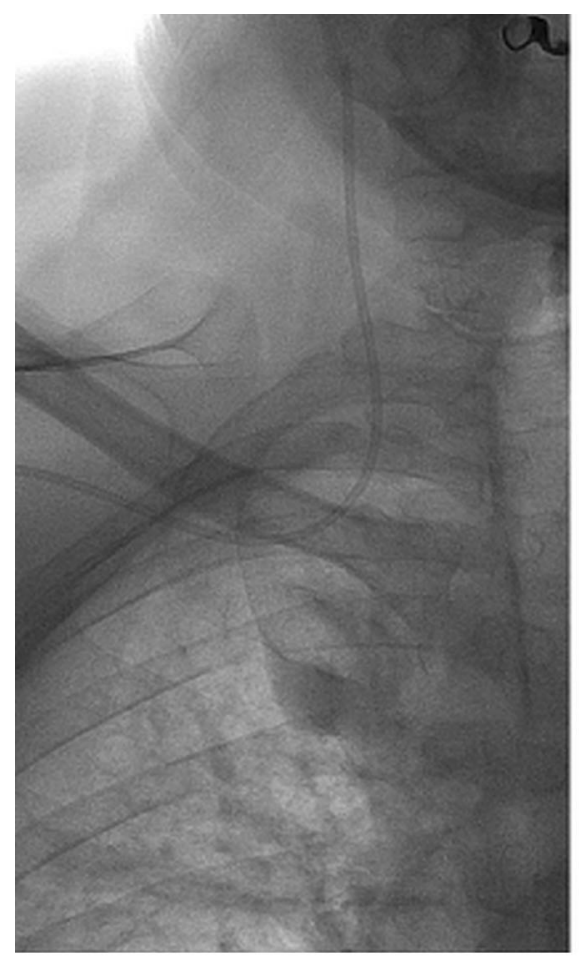

(A)

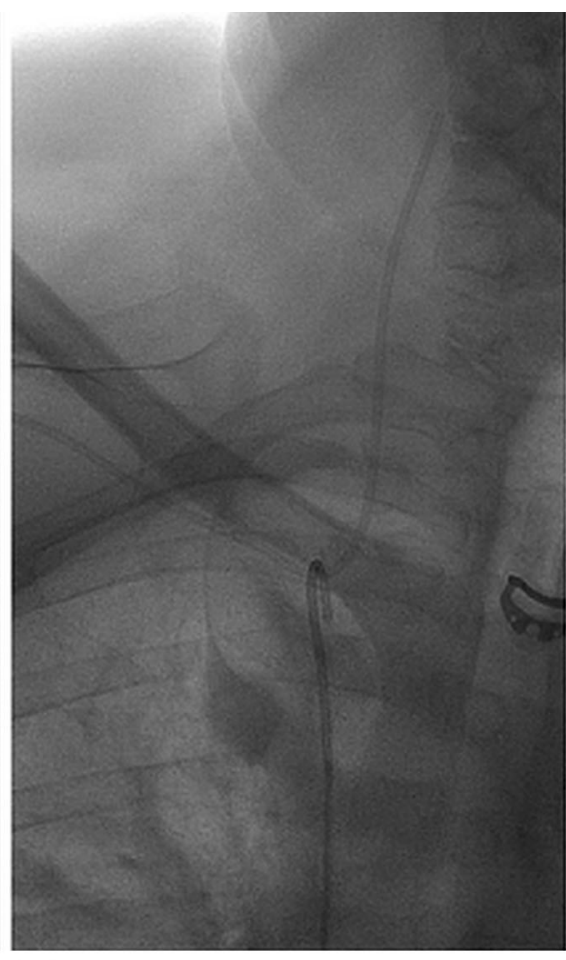

(B)

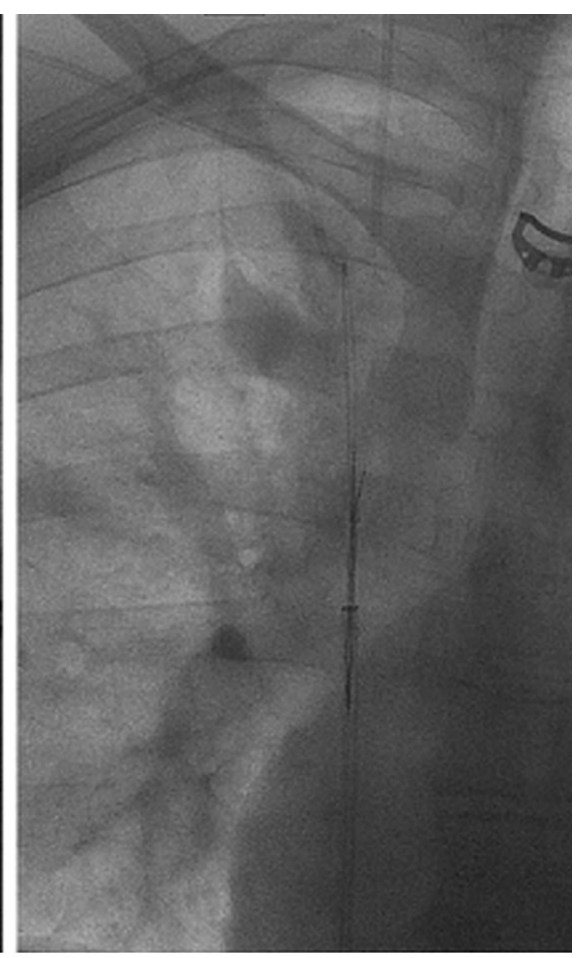

(C)
Fig. 5 Secondary repositioning of a dysfunctional catheter. The patient in these images had a catheter that had migrated in the right internal jugular vein (A). After failed attempts to reposition the catheter using the pigtail (B), we made a loop around the IVAD catheter using a guidewire, which was then grabbed using the snare and pulled downward (C). Regular stripping using the snare catheter was then performed to remove any fibrin sheath (not shown) 


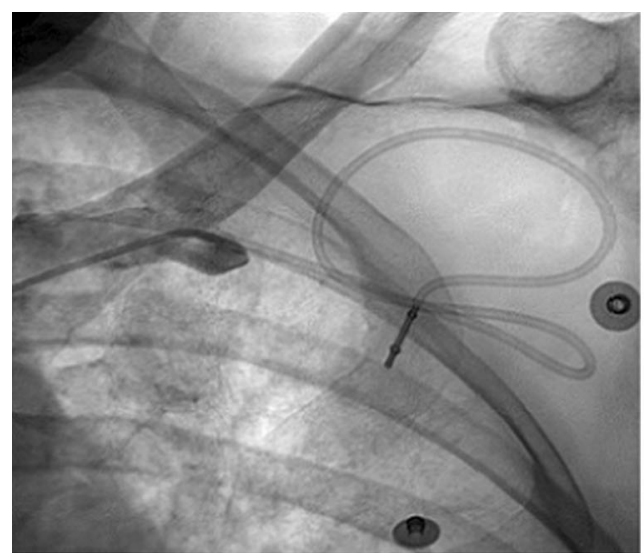

(A)

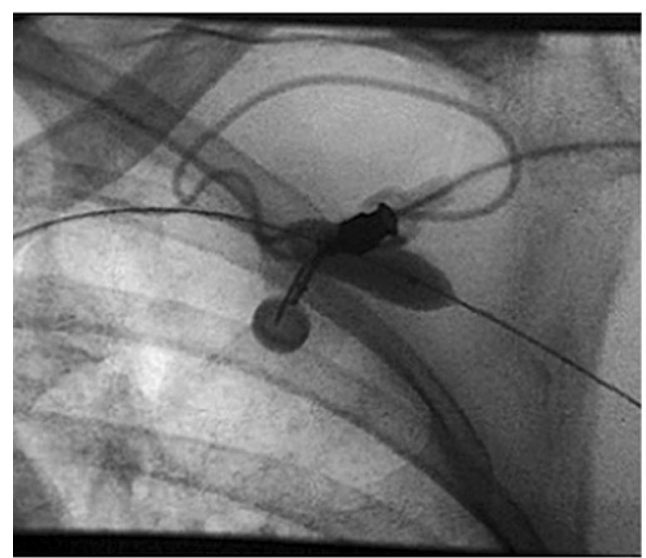

(C)

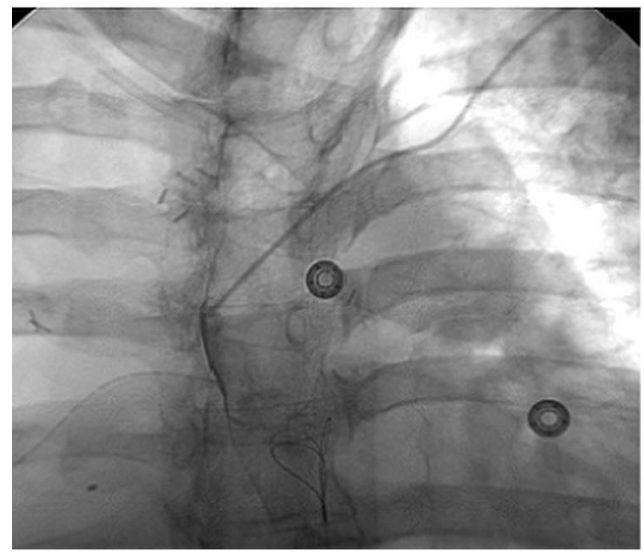

(E)

Fig. 6 Complex case with left subclavian vein stenosis. This case shows an IVAD catheter that was rolled-up in the left subclavian vein. The catheter could not be grabbed due to severe venous stenosis (A). We easily crossed the stenosis with a hydrophilic guidewire (B) and performed balloon angioplasty $(\mathbf{C})$. A clover-shaped snare was then

have described different techniques to mobilize the catheter, sometimes requiring greater gauge venous access [9]. To our knowledge, the mechanical adhesiolysis technique using a pigtail catheter has never been described before. We use a 4F high-torque pigtail catheter because of its

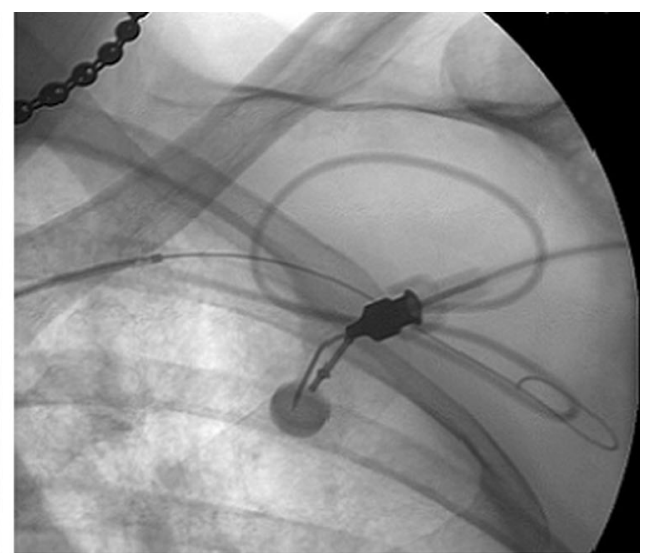

(B)

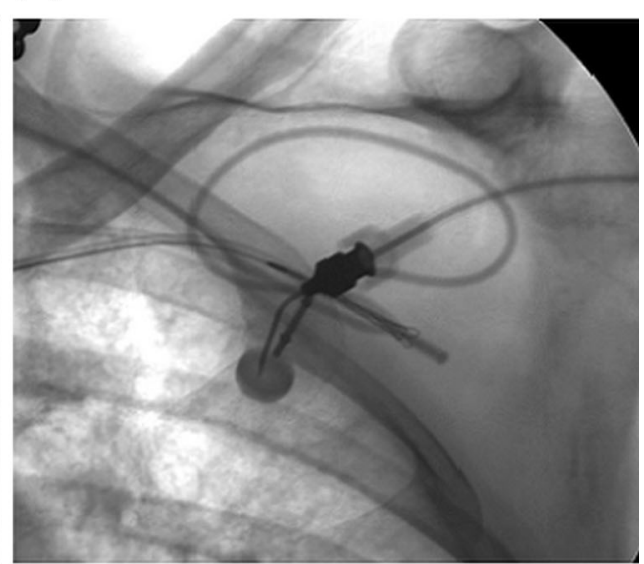

(D)

used to grab the catheter and reposition it in the SVC (D). The final image (E) shows that this catheter is too short and projects into the upper to mid third of the SVC. This is in itself a risk factor for catheter-related complications

stiffer end, but other inverted-curve catheters with different shapes and size can be used as well. Different guidewires can also be placed inside the pigtail to adjust stiffness as needed. In more complex cases, the pigtail can be twisted a couple of times around the IVAD catheter to allow for a 
better grasp and thus facilitate adhesiolysis. This new technique is simple and allows the re-establishment of function in even more catheters than using the snare alone.

Factors that were used to predict vessel wall adherences include catheter tip in contact with the vessel wall, absence of catheter movement with breathing and heart motion, and catheter too short or malpositioned. With experience, we learned to recognize these elements at evaluation, and they were noted in 8 of our patients. In those cases, rather than start the procedure with the snare catheter, we began using the pigtail catheter first to perform the "primary" mechanical adhesiolysis before stripping the catheter with a snare.

When excluding patients who had primary mechanical adhesiolysis, the success rate with snare alone was $82.7 \%$. Using "secondary" mechanical adhesiolysis in those patients turned almost every failed attempt using the snare alone into a technical success. This allowed an increase in success rate up to $88 \%$. When including cases with "primary" mechanical adhesiolysis, the global success rate reached $93.2 \%$. Catheter tip position and important vessel wall adherences were identified as the main causes for salvage failure.

The use of mechanical adhesiolysis was associated with a longer procedure time compared with using the snare alone. Some of the cases were even more complex, requiring additional maneuvers to re-establish flow within catheter and veins. The radiologist's experience could also be a factor influencing procedure length, but this was not evaluated in our study. The range of procedure length is quite wide from $6 \mathrm{~min}$ for simple cases to $107 \mathrm{~min}$ for more complex cases. In addition, we did not notice a shortening of procedure length as the study went on. This shows that each procedure is unique and that every patient case should be addressed with a case-to-case approach.

No complications directly attributed to the procedure were noted. Other similar studies report a low complication rate as well $[8,9,13]$. The fact that we used a smaller introducer sheath could have a protective effect for complications at the site of venous puncture. However, investigators who report using larger sheaths do not necessarily have a greater complication rate. Clinically significant venous rupture or laceration due to catheter stripping or adhesiolysis have not occurred, even in patient who have had their catheter implanted for a long time before the procedures. Given the nature of the procedure itself, we could expect that after stripping part of the material around the catheter would migrate in the pulmonary arteries. Only a few reports in the literature document pulmonary embolisms after catheter manipulation [25-27]. No clinically significant pulmonary embolism was reported in our patients.

In conclusion, catheter tip position is a precipitating factor for IVAD obstruction by fibrin sheath and catheter adherence to vessel wall. The majority of dysfunctional catheters that were evaluated in our series were located in a suboptimal position. Adequate fluoroscopy-guided placement by trained professionals is mandatory when implanting these devices to prevent catheter dysfunction by obstruction.

Our IVAD salvage techniques are safe and efficient. The ability to mobilize the catheter tip from the vessel wall is an important factor for technical success. The introduction of mechanical adhesiolysis using a pigtail catheter allowed for a greater success rate than did using the snare technique alone with no additional risk of complication. In patients who still require long-term use of their IVAD, these procedures can be performed safely to avoid catheter replacement.

Conflict of Interest Stéphane Breault, Frédéric Glauser, Malik Babaker, and Francesco Doenz have no conflict of interest. Salah Dine Qanadli was consultant for Cook Medical Inc. and Cordis Corporation in the last 3 years.

Statement of Informed Consent For this type of study, formal consent is not required.

Statement of Human and Animal Rights This does not apply to this type of study.

\section{References}

1. Yeste Sanchez L, Galbis Caravajal JM, Fuster Diana CA, Moledo Eiras E (2006) Protocol for the implantation of a venous access device (Port-A-Cath System). The complications and solutions found in 560 cases. Clin Transl Oncol 8(10):735-741

2. Walser EM (2012) Venous access ports: Indications, implantation technique, follow-up, and complications. Cardiovasc Intervent Radiol 35(4):751-764

3. Bassi KK, Giri AK, Pattanayak M, Abraham SW, Pandey KK (2012) Totally implantable venous access ports: Retrospective review of long-term complications in 81 patients. Indian J Cancer 49(1):114-118

4. Baskin JL, Pui CH, Reiss U, Wilimas JA, Metzger ML, Ribeiro $\mathrm{RC}$ et al (2009) Management of occlusion and thrombosis associated with long-term indwelling central venous catheters. Lancet 374(9684):159-169

5. Vardy J, Engelhardt K, Cox K, Jacquet J, McDade A, Boyer M et al (2004) Long-term outcome of radiological-guided insertion of implanted central venous access port devices (CVAPD) for the delivery of chemotherapy in cancer patients: institutional experience and review of the literature. Br J Cancer 91:1045

6. Stevens B, Barton SE, Brechbill M, Moenter S, Lou Piel A, Shankle D (2000) A randomized, prospective trial of conventional vascular ports vs. the vortex "clear-flow" reservoir port in adult oncology patients. J Vasc Access Devices 5(2):37-44

7. Gray RJ, Levitin A, Buck D, Brown LC, Sparling YH, Jablonski KA et al (2000) Percutaneous fibrin sheath stripping versus transcatheter urokinase infusion for malfunctioning well-positioned tunneled central venous dialysis catheters: a prospective, randomized trial. J Vasc Interv Radiol 11(9):1121-1129

8. Crain MR, Mewissen MW, Ostrowski GJ, Paz-Fumagalli R, Beres RA, Wertz RA (1996) Fibrin sleeve stripping for salvage of 
failing hemodialysis catheters: technique and initial results. Radiology 198(1):41-44

9. Heye S, Maleux G, Goossens GA, Vaninbroukx J, Jerome M, Stas M (2012) Feasibility and safety of endovascular stripping of totally implantable venous access devices. Cardiovasc Intervent Radiol 35(3):607-612

10. Qanadli SD, Mesurolle B, Sissakian JF, Chagnon S, Lacombe P (2000) Implanted central venous catheter-related acute superior vena cava syndrome: management by metallic stent and endovascular repositioning of the catheter tip. Eur Radiol 10(8):1329-1331

11. Sacks D, McClenny TE, Cardella JF, Lewis CA (2003) Society of interventional radiology clinical practice guidelines. J Vasc Interv Radiol 14(9 Pt 2):S199-S1202

12. Bessoud B, de Baere T, Kuoch V, Desruennes E, Cosset MF, Lassau $\mathrm{N}$ et al (2003) Experience at a single institution with endovascular treatment of mechanical complications caused by implanted central venous access devices in pediatric and adult patients. AJR Am J Roentgenol 180(2):527-532

13. Reddy AS, Lang EV, Cutts J, Loh S, Rosen MP (2007) Fibrin sheath removal from central venous catheters: an internal snare manoeuvre. Nephrol Dial Transplant 22(6):1762-1765

14. Caers J, Fontaine C, Vinh-Hung V, De Mey J, Ponnet G, Oost C et al (2005) Catheter tip position as a risk factor for thrombosis associated with the use of subcutaneous infusion ports. Support Care Cancer 13(5):325-331

15. Cohn DE, Mutch DG, Rader JS, Farrell M, Awantang R, Herzog TJ (2001) Factors predicting subcutaneous implanted central venous port function: the relationship between catheter tip location and port failure in patients with gynecologic malignancies. Gynecol Oncol 83(3):533-536

16. Schutz JC, Patel AA, Clark TW, Solomon JA, Freiman DB, Tuite CM et al (2004) Relationship between chest port catheter tip position and port malfunction after interventional radiologic placement. J Vasc Interv Radiol 15(6):581-587

17. Kuter DJ (2004) Thrombotic complications of central venous catheters in cancer patients. Oncologist 9(2):207-216
18. Nayeemuddin M, Pherwani AD, Asquith JR (2013) Imaging and management of complications of central venous catheters. Clin Radiol 68(5):529-544

19. Funaki B, Szymski GX, Hackworth CA, Rosenblum JD, Burke R, Chang $T$ et al (1997) Radiologic placement of subcutaneous infusion chest ports for long-term central venous access. AJR Am J Roentgenol 169(5):1431-1434

20. Watorek E, Golebiowski T, Letachowicz K, Garcarek J, Kurcz J, Bartosik HA et al (2012) Balloon angioplasty for disruption of tunneled dialysis catheter fibrin sheath. J Vasc Access 13(1): $111-114$

21. Janne d'Othee B, Tham JC, Sheiman RG (2006) Restoration of patency in failing tunneled hemodialysis catheters: a comparison of catheter exchange, exchange and balloon disruption of the fibrin sheath, and femoral stripping. J Vasc Interv Radiol 17(6): 1011-1015

22. Besarab A, Pandey R (2011) Catheter management in hemodialysis patients: delivering adequate flow. Clin J Am Soc Nephrol 6(1):227-234

23. Oliver MJ, Mendelssohn DC, Quinn RR, Richardson EP, Rajan DK, Pugash RA et al (2007) Catheter patency and function after catheter sheath disruption: a pilot study. Clin J Am Soc Nephrol 2(6): 1201-1206

24. Sobolevsky S, Sheiman RG, Faintuch S, Perry L (2007) Femoralto-port through-and-through wire access to reestablish subcutaneous port function. AJR Am J Roentgenol 188(4):1047-1049

25. Brismar B, Hardstedt C, Jacobson S (1981) Diagnosis of thrombosis by catheter phlebography after prolonged central venous catheterization. Ann Surg 194(6):779-783

26. Winn MP, McDermott VG, Schwab SJ, Conlon PJ (1997) Dialysis catheter 'fibrin-sheath stripping': a cautionary tale! Nephrol Dial Transpl 12(5):1048-1050

27. Rockoff MA, Gang DL, Vacanti JP (1984) Fatal pulmonary embolism following removal of a central venous catheter. J Pediatr Surg 19(3):307-309 